\title{
Uma Ontologia de Domínio para a Metodologia de Aprendizagem Baseada em Problemas
}

\author{
Adriana Silva Souza ${ }^{1,4}$, Adolfo Duran ${ }^{2,3}$, Vaninha Vieira ${ }^{1,3}$ \\ ${ }^{1}$ Departamento de Ciência da Computação - Universidade Federal da Bahia (UFBA) \\ Salvador, BA - Brasil \\ ${ }^{2}$ Superintendência de TI (UFBA) - Salvador, BA - Brasil \\ ${ }^{3}$ Centro de Projetos Fraunhofer para Engenharia de Software e Sistemas da UFBA \\ Salvador, BA - Brasil \\ ${ }^{4}$ Instituto Federal da Bahia (IFBA) - Campus Porto Seguro - Porto Seguro, BA - Brazil \\ adriana@ifba.edu.br, adolfo@ufba.br, vaninha@dcc.ufba.br
}

\begin{abstract}
In Eduation, the development of technologies based on ontologies and intelligent agents has been a trend in recent years to aid semantic information processing. New approaches have been proposed to provide an active and dynamic environment for teaching and learning. We observed a lack of ontologies to support specifically the Problem-Based Learning (PBL) methodology. This paper presents a domain ontology that aims to semantically represent the PBL Methodology, in the scope of whiteboard discussions support for tutorial groups. Competency questions, restrictions and SPARQL queries were developed. To evaluate it, a case study was conducted using a real database to check the Ontology consistence, observe the inferences and execute the queries. Results allow to conclude that the proposed ontology reaches its objective.
\end{abstract}

Resumo. Na área de Educação, tem sido uma tendência nos últimos anos o desenvolvimento de tecnologias baseadas em ontologias e agentes inteligentes no auxílio ao processamento de informações semânticas. Novas abordagens visam tornar o processo de ensino e aprendizagem mais dinâmico e ativo. Observamos uma lacuna na proposta de ontologias para o domínio de Aprendizagem Baseada em Problemas (PBL). Esse artigo apresenta uma ontologia de domínio que visa sistematizar e representar semanticamente a metodologia PBL e tem como escopo apoiar a discussão colaborativa das sessões tutoriais. Foram elaboradas questões de competência, que devem ser respondidas pela ontologia, bem como restrições e consultas SPARQL. Para avaliação da ontologia foi realizado um estudo de caso para verificar sua consistência, observar as inferências e executar as consultas a partir de uma base de dados real. Os resultados permitiram concluir que a ontologia proposta atende ao escopo definido.

\section{Introdução}

A Web Semântica idealizada por Berners-Lee (2001) começa a tomar forma com o desenvolvimento de tecnologias baseadas em ontologias e agentes inteligentes, os quais auxiliam no processamento de informações semânticas. Na era da Web 2.0, a integração 
da Web Semântica com a Educação porporciona o desenvolvimento de ambientes de aprendizagem personalizados e novas formas de aprendizagem colaborativa e interativa, contribuindo para um processo de ensino e aprendizagem mais ativo e dinâmico [Kasimati e Zamani 2011]. Ontologias vêm sendo construídas para aplicação ao cenário educacional, afim de promover a interoperabilidade semântica, o compartilhamento e a personalização do conhecimento [Gaeta et al. 2009]. O desenvolvimento de aplicações semânticas apresenta-se como uma tendência na Educação [Martins et al. 2011], possibilitando que novas abordagens ontológicas sejam propostas.

Dentre as metodologias pedagógicas para o processo de construção do conhecimento, podemos destacar a metodologia de Aprendizagem Baseada em Problemas, do inglês Problem-Based Learning (PBL), que se apresenta como uma alternativa à aprendizagem tradicional no desenvolvimento de competências. PBL foi adotada inicialmente nos cursos de Medicina, com boa aceitação. Atualmente, vem sendo utilizada em diversos cursos, mostrando-se eficaz em uma variedade de disciplinas no ensino superior [Hung et al. 2008]. Na metodologia PBL, os estudantes constroem seu próprio conhecimento através da resolução de problemas da vida real. A aprendizagem é centrada no estudante e o conhecimento é adquirido de forma autodirigida. Os estudantes trabalham em grupos colaborativos. O professor é o elemento facilitador do processo e o estudante é o elemento ativo [Ku e Chang 2010].

Na busca por evidências de tecnologias da Web Semântica para a metodologia PBL, foi realizado um mapeamento sistemático [Souza et al. 2014], visando descobrir quais ontologias foram propostas para o domínio PBL. Essa pesquisa observou que existem poucas formalizações utilizando linguagem ontológica nesse domínio. Com o objetivo de apresentar formalmente os conceitos relacionados a essa metodologia e permitir o compartilhamento, reuso e evolução da ontologia pela comunidade, esse artigo apresenta uma ontologia de domínio para a metodologia de Aprendizagem Baseada em Problemas. Seu escopo é apoiar as discussões colaborativas que acontecem nas sessões tutoriais, uma das etapas da PBL no processo de resolução do problema.

O presente artigo está organizado da seguinte forma: a Seção 2 oferece uma visão geral sobre PBL. A Seção 3 discute os trabalhos relacionados. A Seção 4 apresenta a ontologia proposta e a metodologia utilizada. A Seção 5 discute o processo de avaliação e os resultados obtidos. A Seção 6 conclui e apresenta os trabalhos futuros.

\section{Aprendizagem Baseada em Problemas}

A metodologia PBL surgiu no final dos anos 60 no curso de Medicina da Universidade de McMaster, Canadá [Barrows 1996]. No Brasil, PBL foi adotada em alguns cursos de Medicina e tem sido empregada em diversas áreas do ensino superior, como Pedagogia [Esteves 2006], Administração de Empresas [Stinson e Milter 1996], Engenharias [Ribeiro 2005], dentre outras. PBL é uma metodologia inspirada no construtivismo. Seu objetivo é estimular os estudantes a desenvolver o pensamento crítico, habilidades para resolução de problemas, aprendizagem autônoma, capacidade de comunicação e colaboração. Para isso, usa a resolução de problemas reais, muitas vezes complexos, que podem não possuir uma solução única e, muitas vezes, não trivial [Sousa 2011].

Uma referência bastante popular para sistematização da PBL é o referencial dos "sete passos" proposto pela Universidade de Maastricht na década de 70 [Deelman e Hoeberigs 2009]. Outra referência clássica é o Ciclo PBL proposto por Hmelo-Silver 
(2004). A Figura 1 apresenta um framework conceitual, proposto nessa pesquisa, que visa integrar essas duas definições clássicas, consideradas complementares.

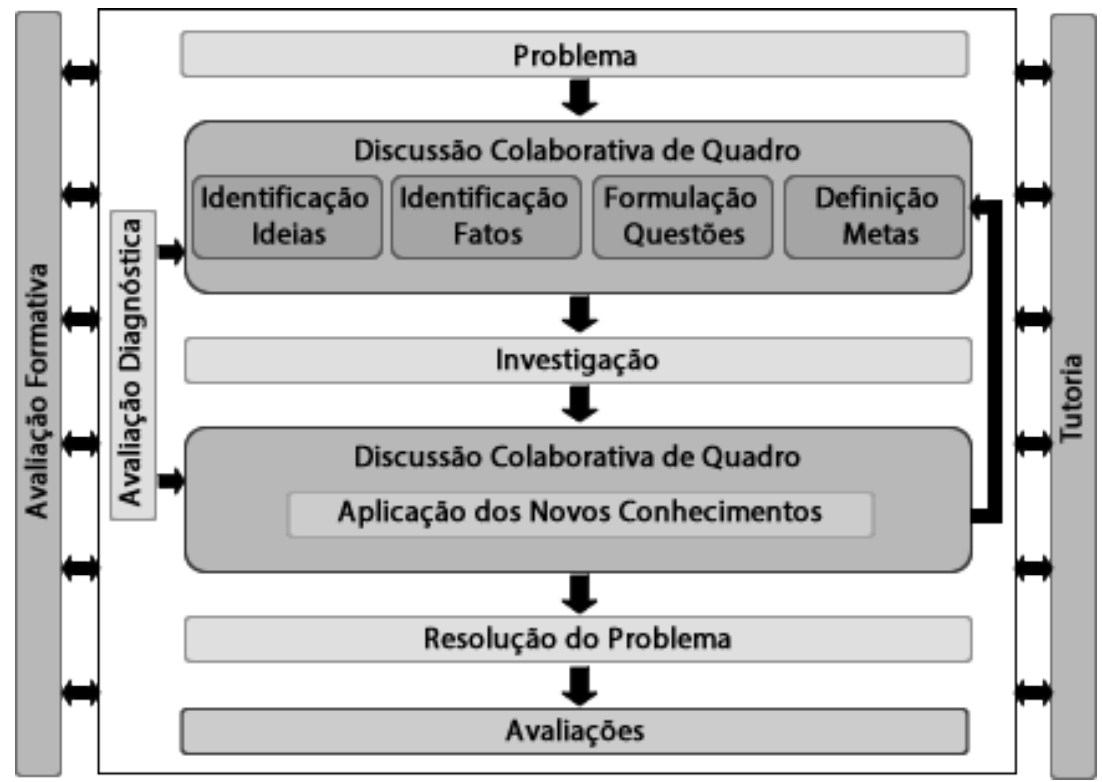

Figura 1. Framework conceitual da metodologia PBL (Elaborado pelos autores)

A sessão PBL começa com a apresentação de um problema aos membros do grupo, onde é levantado o cenário do problema. Após a apresentação do problema, os estudantes fazem uma discussão colaborativa de quadro. Nessa etapa, identificam ideias e fatos, formulam questões e definem metas de aprendizagem. Esse conceitual levantado é geralmente registrado em um quadro branco. A seguir, os estudantes seguem para o estudo autônomo, onde investigam conteúdos que auxiliam nas respostas às questões e metas definidas. Na próxima etapa, os estudantes se reunem, novamente, em uma sessão tutorial onde é realizada uma nova discussão colaborativa de quadro para aplicação dos novos conhecimentos [Hmelo-Silver 2004]. Novas ideias, fatos, questões e metas podem ser identificados, formando um ciclo de interações. Ao término da resolução de cada problema, acontece o processo de avaliação. Os estudantes refletem sobre o conhecimento adquirido, avaliam o processo, o tutor, os pares e se auto-avaliam. Para que a metodologia seja eficaz é necessário que haja uma tutoria do processo pelo tutor, que ele observe cuidadosamente as sessões, fazendo avaliação diagnótica para identificar as deficiências dos estudantes, desenvolvendo estratégias específicas para superar as deficiências detectadas. Durante esse processo, é necessário também, que seja realizada a avaliação formativa que analisa o desenvolvimento das competências desejadas [Hmelo-Silver 2006].

\section{Trabalhos Correlatos}

Conforme reportado em estudo de mapeamento sistemático realizado [Souza et al. 2014], a literatura apresenta poucas propostas de ontologias de apoio a PBL. Algumas dessas abordagens são apresentadas a seguir.

Aparicio et al. (2012) propõem uma interface Web que utiliza técnicas de web semântica para melhorar a experiência do estudante no processo de aprendizagem. $\mathrm{O}$ sistema proposto usa uma ontologia de termos para auxiliar a busca por termos médicos, permitindo a inserção de um texto da área médica, a fim de fundir as fontes de 
conhecimento e torná-las disponíveis para os estudantes. O sistema compila, em listas, um conjunto de conceitos médicos extraídos da ontologia Freebase ${ }^{1}$.

Fontes et al. (2011) apresentam uma ontologia para solucionar o problema relacionado à falta de representação formal dos conceitos inerentes a PBL buscando facilitar o acesso a informações do domínio. A ontologia proposta também objetiva verificar se os estudantes estão cumprindo o ciclo PBL.

Sudhana et al. (2013) propõem um modelo de arquitetura para sistemas virtuais de aprendizagem sensível e adaptado ao contexto do estudante e um framework baseado em ontologias para pesquisa e entrega de conteúdos adaptados ao contexto do estudante.

Kazi et al. (2013) propõem um sistema de tutoria inteligente para a área médica baseado em ontologia para reduzir a sobrecarga de conhecimento. Os conceitos e termos médicos são definidos em um domínio ontológico. A ontologia permite aos estudantes formular suas próprias hipóteses e soluções, a partir da seleção dos conceitos.

O trabalho de Fontes et al. (2011) é o que mais se aproxima da nossa proposta. No entanto, nossa ontologia difere em alguns aspectos, a saber: (1) foi construída seguindo uma metodologia de construção de ontologias; (2) foram definidas questões de competência, criadas consultas SPARQL e realizado um estudo de caso, permitindo avaliar a ontologia de acordo com as questões definidas e as inferências observadas, enquanto a ontologia de Fontes et al. (2011) apresenta apenas classes e propriedades e nenhum estudo de avaliação; (3) o reuso da ontologia de Fontes et al. (2011) não foi possível, devido o autor não ter compartilhado a ontologia em nenhum repositório e não possui-la mais; nossa ontologia foi implementada do ponto inicial, tendo Fontes et al. (2011) como referência, e será disponibilizada para reuso da comunidade.

\section{Uma Ontologia de Apoio à Metodologia PBL}

Para desenvolvimento da ontologia, foi feita uma análise da literatura sobre os termos relevantes para o domínio de PBL. A formalização proposta adota a referência dos sete passos da Universidade de Maastricht [Deelman e Hoeberigs 2009], a representação do quadro branco e a definição do processo tutorial PBL definida por Hmelo-Silver (2004).

Essa ontologia de domínio tem por objetivo descrever os elementos essenciais que devem ser considerados na Aprendizagem Baseada em Problemas, visando representá-los semanticamente. Além disso, busca apoiar as sessões tutoriais, por meio de consultas que detectam dificuldades dos estudantes na discussão colaborativa, como: questões de aprendizagem que se repetem na resolução de um prolema; estudantes que não foram designados para funções como coordenador; dentre outras.

Para construir a ontologia, utilizou-se o método 101 [Noy e Mcguiness 2001]. A ontologia foi implementação com a ferramenta Protégé 4.3, por ser popular, de código aberto, de fácil utilização e interoperabilidade [Noy et al. 2000], além de comportar o desenvolvimento da ontologia proposta, sem maiores restrições. A linguagem de descrição escolhida foi a OWL (Web Ontology Language) por ser uma linguagem robusta e recomendada pela W3C (World Wide Web Consortium), principal organização de padronização da web. A linguagem definida para realização de consultas na

\footnotetext{
1 http://www.freebase.com.
} 
ontologia foi a SPARQL, por ser também suportada pelo Protégé, recomendada pela W3C e candidata a linguagem padrão para uso na web semântica.

A ontologia implementada está estruturada da seguinte maneira: (i) classes e subclasses, que representa a taxonomia do domínio e abrange um conjunto de classes e sua respectiva hierarquia; (ii) propriedades, que podem ser de dois tipos, Object Property (qualificam ou relacionam as classes) e Datatype Property (representam atributos das classes); (iii) instâncias, que representam indivíduos das classes; (iv) restrições representam afirmações lógicas das quais outras afirmações podem ser inferidas; e (v) consultas SPARQL, que realizam as consultas na ontologia.

\subsection{Domínio, Escopo e Questões de Competência}

O primeiro passo no desenvolvimento de uma ontologia, segundo o método 101, é a definição do domínio e escopo a ser tratado. O domínio da ontologia proposta é a representação e formalização semântica da metodologia PBL. O escopo é apoiar a discussão colaborativa, por meio da detecção de questões de aprendizagem que os estudantes possuem dificuldade para solucionar, metas não cumpridas e estudantes não designados para funções especiais nas sessões PBL. As questões de competência são questionamentos que a ontologia deve ser capaz de responder. Estas, além de justificar a existência da ontologia, servem para sua futura avaliação. As seguintes questões foram definidas, com base no escopo da ontologia:

- QC1: Que questões de aprendizagem os alunos têm dificuldade de solucionar?

- QC2: Quais as metas que não foram cumpridas?

- QC3: Quais estudantes não foram designados para coordenador de sessão?

- QC4: Quais estudantes não foram designados para secretário de mesa?

- QC5: Quais estudantes não foram designados para secretário de quadro?

\subsection{Classes, Propriedades e Restrições}

Os conceitos da ontologia foram definidos no idioma inglês, para permitir maior visibilidade, reuso e extensão da mesma. Conforme ilustrado na Figura 2, a Ontologia de domínio PBL possui uma superclasse Process, que representa o processo PBL, e possui 6 classes: Problem, TutorialGroup, TutorialSession, SelfStudy, SkillCompetence e StageAbstraction. Essas, por sua vez, possuem subclasses, como descrito a seguir.

A classe Problem representa um problema que será trabalhado no processo PBL. Esse problema é uma situação real, ou potencialmente real, passível de ser encontrada futuramente na vida profissional dos estudantes. Ele visa cobrir uma dada área de conteúdos, objetivando construção de conhecimento, habilidades e competências. Essa classe possui três subclasses (LearningGoal, ProblemScenario e EvaluationProblem). LearningGoal representa os objetivos de aprendizagem do problema. ProblemScenario representa o cenário do problema, os termos conhecidos (subclasse KnownTerm) e os termos desconhecidos (subclasse UnknownTerm). As subclasses KnownTerm e UnknownTerm são disjuntas. EvaluationProblem representa a avaliação realizada ao final da resolução de cada problema, e considera: cumprimento das metas, assiduidade, colaboração e comportamento, além da avaliação dos estudantes como coordenador, secretário de quadro e secretário de mesa e as avaliações referentes à entrega do produto final e relatório final de resolução do problema. 


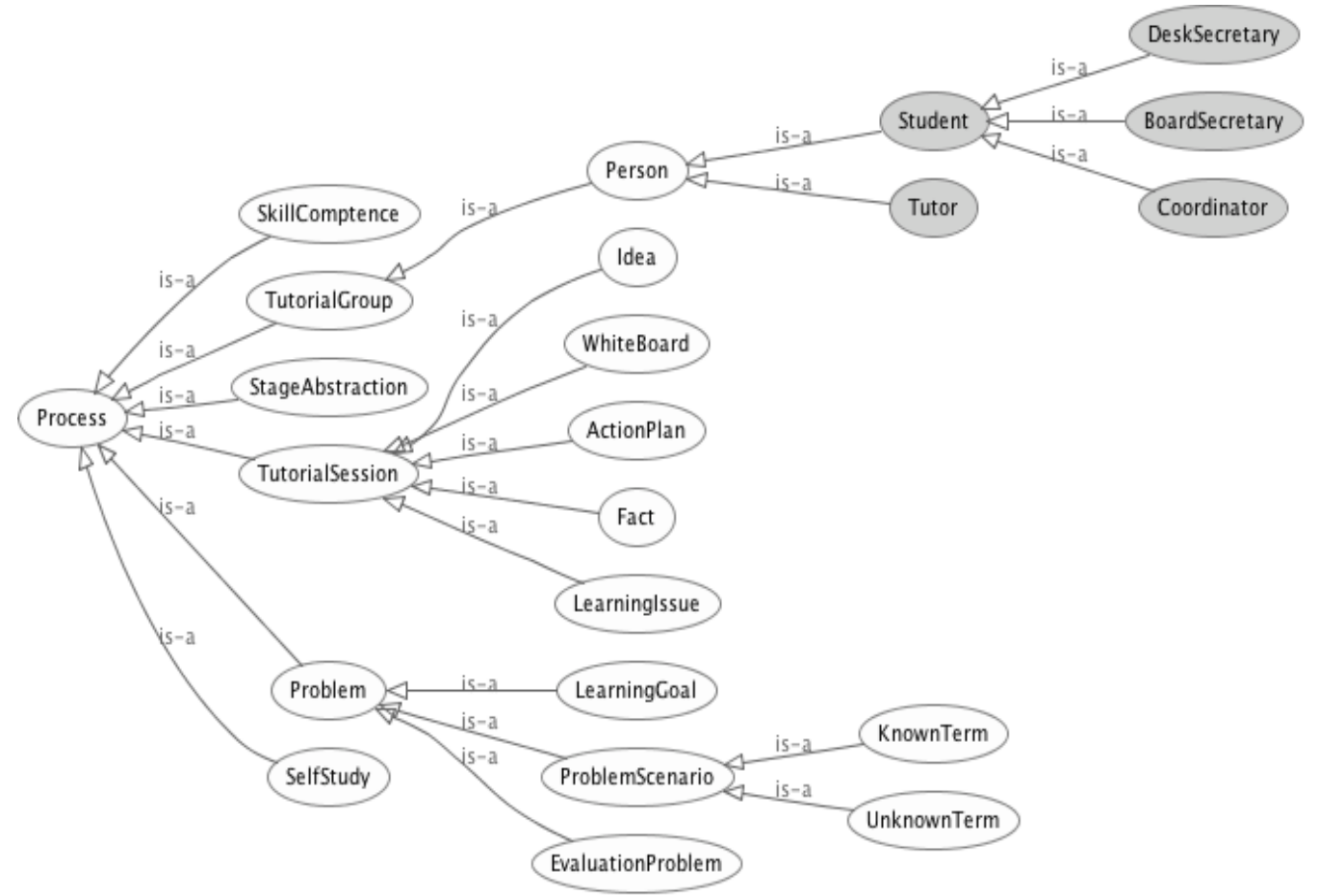

Figura 2: Hierarquia das classes da Ontologia PBL

A classe TutorialGroup representa o grupo tutorial PBL, composto por um tutor (classe Tutor) e estudantes (classe Student), que são entre 8 a 10 estudantes, de acordo com a PBL clássica. As classes Tutor e Student são disjuntas. Os estudantes podem assumir funções especiais nas sessões tutoriais. O secretário de quadro, representado pela classe BoardSecretary, é o responsável por registrar as discussões colaborativas no quadro branco. O secretário de mesa, representado pela classe DeskSecretary, é o responsável por gerar o relatório da sessão e enviar para o tutor e demais estudantes. $\mathrm{O}$ coordenador, representado pela classe Coordinator, coordena a sessão e as discussões.

A classe TutorialSession representa reuniões realizadas pelo grupo tutorial para discutir o problema proposto. Ela possui cinco subclasses (WhiteBoard, Idea, Fact, LearningIssue e ActionPlan). WhiteBoard representa o registro e a evolução das discussões da sessão tutorial, realizadas em um quadro branco. O quadro branco é uma união disjunta das subclasses: Idea (associação livre de ideias relacionadas ao problema), Fact (levantamento do que se conhece sobre o problema), LearningIssue (levantamento de questões ou dúvidas que o grupo não sabe responder sobre o problema) e ActionPlan (definição do que será necessário fazer para aprofundar os conhecimentos incompletos levantados na discussão visando a resolução do problema).

A classe SelfStudy representa a fase de estudo autônomo, onde os estudantes pesquisam na literatura conteúdos que os auxiliarão a fornecer respostas às questões de aprendizagem e cumprimento das metas. A classe SkillCompetence representa as competências e habilidades desenvolvidas ou que a PBL pode desenvolver. A classe StageAbstraction representa a fase de abstração, que é a última etapa,, onde ocorre a avaliação de todo o processo.

Após a definição das classes da ontologia, foram descritas as estruturas dessas classes: propriedades, relacionamentos e restrições. Foram especificadas 27 propriedades do tipo ObjectProperty (que representam relacionamentos entre dois 
indivíduos ou classes) e 25 propriedades DataProperty (que representam atributos das classes e relacionam uma característica de um indivíduo a um tipo de dado abstrato. A listagem dessas propriedades são exibidas na Figura 3. Por limitação de espaço tais propriedades não serão detalhadas nesse artigo.

\begin{tabular}{|c|c|c|}
\hline & $\begin{array}{l}\text { topObjectProperty } \\
\text { hasActionPlan } \\
\text { hasBoardSecretary } \\
\text { hasCoordinatorSession } \\
\text { hasDeskSecretary } \\
\text { hasEvaluationProblem } \\
\text { hasFact } \\
\text { hasIdea } \\
\text { hasKnownTerm } \\
\text { hasLearningGoal } \\
\text { hasLearningIssue } \\
\text { hasPerson } \\
\text { hasProblem } \\
\text { hasProblemScenario } \\
\text { hasProcess } \\
\text { hasSelfStudy } \\
\text { hasStageAbstraction } \\
\text { hasTutor } \\
\text { hasTutorialGroup } \\
\text { hasTutorialSession } \\
\text { hasUnknownTerm } \\
\text { hasWhiteBoard } \\
\text { isAdviseMetting } \\
\text { isBoardSecretary } \\
\text { isCoordinatorSession } \\
\text { isDeskSecretary } \\
\text { isDevelopSkillCompetence } \\
\text { isEvaluated }\end{array}$ & $\begin{array}{l}\nabla \text { topDataProperty } \\
\text { achievementOfGoals } \\
\text { assiduity } \\
\text { behavior } \\
\text { boardSecretaryEvaluation } \\
\text { code } \\
\text { collaboration } \\
\text { coordinatorEvaluation } \\
\text { descriptionProblem } \\
\text { deskSecretaryEvaluation } \\
\text { email } \\
\text { finalProduct } \\
\text { goal } \\
\text { ideaDescription } \\
\text { learningObjectives } \\
\text { learningQuestion } \\
\text { name } \\
\text { pairEvaluation } \\
\text { priorKnowledge } \\
\text { reportEvaluation } \\
\text { selfEvaluation } \\
\text { semester } \\
\text { SessionDate } \\
\text { sessionNumber } \\
\text { term } \\
\text { tutorEvaluation }\end{array}$ \\
\hline
\end{tabular}

Figura 3: Propriedades da Ontologia PBL

As restrições podem ajudar a definir os indivíduos de uma classe e são utilizadas pelo motor de inferência para validar a hierarquia e consistência das classes. Foram definidas 10 restrições para garantir os conceitos e a consistência da ontologia. A Figura 4 apresenta a restrição criada para definir um indivíduo da classe Tutor.

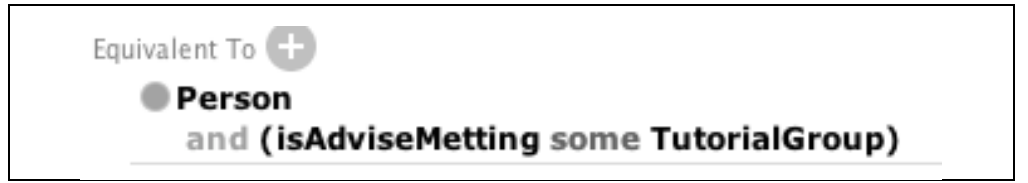

Figura 4: Restrição da Classe Tutor

A restrição da Figura 4 define que os indivíduos da classe Tutor são membros da classe Person e tutora algum grupo tutorial e a condição de ser pessoa e tutorar algum grupo é condição suficiente para ser um indivíduo da classe Tutor.

\section{Avaliação da Ontologia}

Para avaliar a ontologia, foi realizado um estudo de caso em um curso de Engenharia da Computação que utiliza a metodologia PBL. Três turmas foram selecionadas e observadas durante aproximadamente três meses (Março a Maio de 2014), recolhendo informações para instanciar na ontologia. As observações foram realizadas em três disciplinas diferentes: $2^{\circ}$ semestre PBL de Programação, $4^{\circ}$ semestre PBL de Conectividade e Concorrência e $7^{\circ}$ semestre PBL de Processamentos Digitais de Sinais. 
Inicialmente, buscou-se observar se o processo PBL definido na ontologia estava de acordo com a prática. Durante as observações e entrevistas com tutores e especialistas surgiram novos termos, como por exemplo, avaliação do problema, secretário de quadro, secretário de mesa, que foram acrescentados à ontologia.

Em um segundo momento, as observações visaram a obtenção de informações reais para definir as instâncias da ontologia. Foram instanciados três processos PBL (disciplinas) e seus respectivos problemas, grupos, sessões, quadro branco, resultando em um total de 30 sessões PBL. Essa base de dados real possibilitou a realização de testes de consistência da ontologia, com a execução do motor de inferência Pellet e a realização das consultas SPARQL para responder às questões de competência (ver Tabela 1). Nos testes de consistência, verificou-se: a classificação da taxonomia, avaliando se as classes inferidas estavam de acordo com a hierarquia proposta; os tipos inferidos, identificando se as restrições e regras semânticas estavam corretamente definidas. Os primeiros testes foram realizados à medida que as instâncias foram sendo criadas e as falhas foram corrigidas à medida que foram sendo detectadas.

Para responder às questões de competência $\mathrm{QC} 1$ e QC2, partimos do pressuposto que as questões e metas que aparecem mais de uma vez no quadro branco indicam dificuldades para resolvê-las. Para responder essas questões, as consultas SPARQL ilustradas na Tabela 1 foram criadas e testadas na ontologia. Ao integrar a ontologia numa aplicação, essas consultas podem auxiliar tutores na identificação das dificuldades dos estudantes. Num contexto de recomendação de conteúdos, a aplicação poderá indicar objetos de aprendizagem a partir dessa questões.

Tabela 1. Consultas SPARQL da Ontologia PBL

\begin{tabular}{|c|c|}
\hline Código & Consulta \\
\hline QC1 & $\begin{array}{l}\text { SELECT ?question (COUNT (?question) AS ?total) WHERE } \\
\text { \{?LearningIssue pbl:LearningQuestion ?question.\} } \\
\text { GROUP BY ?question HAVING (COUNT(?question)>1) }\end{array}$ \\
\hline QC2 & $\begin{array}{l}\text { SELECT ?goal (COUNT (?goal) AS ?total) WHERE } \\
\text { \{?Action pbl: Action ?goal. } \\
\text { GROUP BY ?goal HAVING (COUNT (?goal)>1) }\end{array}$ \\
\hline QC3 & $\begin{array}{l}\text { SELECT ?Grupo ?Aluno Where } \\
\text { \{?Grupo pbl:hasPerson ?Aluno. MINUS \{ } \\
\text { ?Sessao pbl:hasCoordinatorSession?Aluno\} } \\
\text { ORDER BY (?Grupo) (?Aluno) }\end{array}$ \\
\hline QC4 & $\begin{array}{l}\text { SELECT ?Grupo ?Aluno Where } \\
\text { \{?Grupo pbl:hasPerson ?Aluno. MINUS \{?Sessao } \\
\text { pbl:hasDeskSecretary?Aluno\}\} } \\
\text { ORDER BY (?Grupo) (?Aluno) }\end{array}$ \\
\hline QC5 & $\begin{array}{l}\text { SELECT ?Grupo ?Aluno Where } \\
\text { \{?Grupo pbl:hasPerson ?Aluno. MINUS \{?Sessao pbl: hasBoardSecretary } \\
\text { ?Aluno\} } \\
\text { ORDER BY (?Grupo) (?Aluno) }\end{array}$ \\
\hline
\end{tabular}

Para responder às questões QC3, QC4 e QC5 foram criadas e avaliadas as consultas SPARQL ilustradas na Tabela 1. Os resultados retornaram os estudantes que não foram coordenadores, secretário de mesa e secretário de quadro, respectivamente. Ao integrar a ontologia com uma aplicação, essas consultas podem auxiliar o tutor a identificar estudantes que ainda não assumiram as respectivas funções nas sessões tutoriais e recomendar estudantes para serem coordenadores e secretários. 


\section{Conclusão e Trabalhos Futuros}

Esse artigo apresentou uma ontologia para representação semântica da metodologia de Aprendizagem Baseada em Problemas (PBL). Foram considerados os conceitos e aspectos fundamentais e relevantes da metodologia a partir de um levantamento na literatura. A ontologia proposta representa um avanço em relação a trabalhos existentes, pois apoia a discussão colaborativa da sessão PBL, a partir da detecção de questões e metas recorrentes na discussão de quadro branco e identificação de estudantes que não foram designados para funções definidas na metodologia. Além disso, apresenta uma formalização semântica para PBL usando como base duas referências clássicas. Outra vantagem é a possibilidade de reuso em diversas aplicações desenvolvidas para PBL.

Como trabalhos futuros, a ontologia será ampliada com novas questões de competências e consultas. Uma avaliação com especialistas será realizada para saber se as classes e propriedades definidas são suficientes para modelar aplicações PBL, a ontologia também será integrada a uma aplicação e um novo estudo de caso será realizado para avaliação da mesma.

\section{Agradecimentos}

Os autores agradecem o apoio recebido para realização dessa pesquisa do Centro de Projetos Fraunhofer para Engenharia de Software e Sistemas da UFBA, uma parceria conjunta da Sociedade Fraunhofer com a Universidade Federal da Bahia, com apoio do Governo do Estado da Bahia.

\section{Referências}

Aparicio, F., De Buenaga, M., Rubio, M., \& Hernando, A. (2012). An intelligent information access system assisting a case based learning methodology evaluated in higher education with medical students. Computers \& Education, 58(4), 1282-1295.

Barrows, H. S. (1996). Problem-based learning in medicine and beyond: A brief overview. New directions for teaching and learning, (68), 3-12.

Berners-Lee, T., Hendler, J., \& Lassila, O. (2001). The Semantic Web. Scientific American. v. 284, n. 5, p. 28-37.

Deelman, A., \& Hoeberigs, B. (2009). A ABP no contexto da universidade de Maastricht. Aprendizagem baseada em problemas no ensino superior. Ulisses F. Araúlo, Genoveva Sastre (org.). São Paulo, Brasil: Summus, 79-100.

Esteves, E. (2006). O ensino da Física e Química através da Aprendizagem Baseada na Resolução de Problemas: um estudo com futuros professores sobre concepções e viabilidade. In Actas do Congresso Internacional Aprendizaje Baseado en Porblemas $(P B L-A B P)$. Lima, Perú: Universidade Pontificia Católica.

Fontes, L. M. D. O., Mendes Neto, F. M., \& Pontes, A. Á. A. (2011). OntoPBL: Uma Ontologia de Domínio sobre Aprendizagem Baseada em Problema. In Anais do Simpósio Brasileiro de Informática na Educação (Vol. 1, No. 1).

Gaeta, M., Orciuoli, F., \& Ritrovato, P. (2009). Advanced ontology management system for personalised e-learning. Knowledge-Based System., v. 22, p. 292-301. 
Hmelo-Silver, C. E. (2004). Problem-based learning: What and how do students learn?. Educational Psychology Review, 16(3), 235-266.

Hmelo-Silver, C. E., \& Barrows, H. S. (2006). Goals and strategies of a problem based learning facilitator. Interdisciplinary Journal of Problem-based Learning, 1(1), 4.

Hung, W., Jonassen, D. H., \& Liu, R. (2008). Problem-based learning. Handbook of research on educational communications and technology, 3, 485-506.

Kasimati, A., \& Zamani, E. (2011). Education and learning in the Semantic Web, In Informatics (PCI), 15th Panhellenic Conference on (pp. 338-344). IEEE.

Kazi, H., Haddawy, P., \& Suebnukarn, S. (2013). Clinical reasoning gains in medical PBL: an UMLS based tutoring system. Journal of Intelligent Information Systems, 116.

Ku, D. T., \& Chang, C. S. (2010). A framework of PBL strategy integrated in LMS and a ubiquitous learning environment. In Networked Computing and Advanced Information Management (NCM), Sixth International Conference on (pp. 722-726). IEEE.

Martin, S., Diaz, G., Sancristobal, E., Gil, R., Castro, M., \& Peire, J. (2011). New technology trends in education: Seven years of forecasts and convergence. Computers \& Education, 57(3), 1893-1906.

Noy, N., Fergerson, R., \& Musen., M. (2000). The knowledge model of Protege-2000: Combining interoperability and flexibility. 2th International Conference on Knowledge Engineering and Knowledge Management (EKAW'2000), Juan-les-Pins, France.

Noy, N.F., \& Mcguiness, D.L. (2001). Ontology Development 101: A Guide to Creating your first ontology. Stanford Knowledge Systems Laboratory Technical Report KSL-01-05 and Stanford Medical Informatics Technical Report SMI-20010880 .

Ribeiro, L. R. C. (2005). A aprendizagem baseada em problemas (PBL): uma implementação na educação em engenharia na voz dos atores". Tese de Doutorado (Educação). Universidade Federal de São Carlos.

Sousa, S. D. O. (2011). Aprendizagem Baseada em Problemas (PBL - Problem-Based Learning): Estratégia para o Ensino e Aprendizagem de Algoritmos e Conteúdos Computacionais. Nuances: Estudos sobre Educação, 18(19).

Souza, S. A., Duran, A., \& Vieira, V. (2014). Um estudo de mapeamento sistemático sobre ontologias para a metodologia de aprendizagem baseada em problemas. In Anais do Simpósio Brasileiro de Informática na Educação. XXV SBIE.

Stinson, J. E., \& Milter, R. G. (1996). Problem-based learning in business education: Curriculum design and implementation issues. New directions for teaching and learning, (68), 33-42.

Sudhana, K., Raj, V. C., \& Suresh, R. M. (2013). An ontology-based framework for context-aware adaptive e-learning system. In Computer Communication and Informatics (ICCCI), 2013 International Conference on (pp. 1-6). IEEE. 